\title{
Differential Diagnosis of Flavivirus Infections in Horses Using Viral Envelope Protein Domain III Antigens in Enzyme-Linked Immunosorbent Assay
}

\author{
Thisun B.H. Piyasena,, ${ }^{1, *}$ Yin X. Setoh, ${ }^{1, *}$ Jody Hobson-Peters, ${ }^{1}$ Natalie A. Prow, Helle Bielefeldt-Ohmann, ${ }^{1,2}$ \\ Alexander A. Khromykh, David Perera, Mary J. Cardosa, ${ }^{3}$ Peter D. Kirkland, and Roy A. Hall ${ }^{1}$
}

\begin{abstract}
In Australia, infection of horses with the West Nile virus (WNV) or Murray Valley encephalitis virus (MVEV) occasionally results in severe neurological disease that cannot be clinically differentiated. Confirmatory serological tests to detect antibody specific for MVEV or WNV in horses are often hampered by cross-reactive antibodies induced to conserved epitopes on the envelope (E) protein. This study utilized bacterially expressed recombinant antigens derived from domain III of the E protein (rE-DIII) of MVEV and WNV, respectively, to determine whether these subunit antigens provided specific diagnostic markers of infection with these two viruses. When a panel of 130 serum samples, from horses with known flavivirus infection status, was tested in enzyme-linked immunosorbent assay (ELISA) using rE-DIII antigens, a differential diagnosis of MVEV or WNV was achieved for most samples. Time-point samples from horses exposed to flavivirus infection during the 2011 outbreak of equine encephalitis in south-eastern Australia also indicated that the rE-DIII antigens were capable of detecting and differentiating MVEV and WNV infection in convalescent sera with similar sensitivity and specificity to virus neutralization tests and blocking ELISAs. Overall, these results indicate that the rE-DIII is a suitable antigen for use in rapid immunoassays for confirming MVEV and WNV infections in horses in the Australian context and warrant further assessment on sensitive, high-throughput serological platforms such as multiplex immune assays.
\end{abstract}

Keywords: equine disease, flavivirus, Murray Valley encephalitis virus, serology, viral envelope domian III, West Nile virus

\section{Introduction}

$\mathbf{W}$ EST NILE VIRUS (WNV) and Murray Valley encephalitis virus (MVEV) are mosquito-borne flaviviruses that are endemic to the tropical north of Australia. Although the Australian strain of WNV shares high antigenic similarity with strains of WNV circulating in other parts of the world (Hall 2000, Scherret et al. 2001), disease is infrequently observed. MVEV is the leading cause of arboviral encephalitis in humans in Australia (Mackenzie and Williams 2009, Schweitzer et al. 2009, Knox et al. 2012) and infection with either virus can produce equine disease with clinical symptoms such as ataxia, altered mental state, and encephalitis, which can subsequently lead to permanent neurological sequelae or death (Roche et al. 2013, Barton et al. 2015).

MVEV and WNV activity is monitored in Australia by sero-conversion in sentinel chickens and by virus isolation from captured Culex annulirostris mosquitoes, which is the main transmission vector for these viruses (Mackenzie et al. 1994, Bolisetty et al. 2002). Occasionally, these viruses spread from their northern endemic foci to more southerly regions during times of unusually high rainfall (Mackenzie et al. 1994, Brown et al. 2002, Hall et al. 2002, Mackenzie and Williams 2009).

In 2011, there was an outbreak of equine viral encephalitis in south-eastern Australia with at least 1000 documented

\footnotetext{
${ }^{1}$ Australian Infectious Diseases Research Centre, School of Chemistry and Molecular Biosciences, The University of Queensland, St Lucia, Australia.

${ }^{2}$ School of Veterinary Science, University of Queensland, Gatton, Australia.

${ }^{3}$ Institute of Health \& Community Medicine, Universiti Malaysia Sarawak, Kota Samarahan, Malaysia.

${ }^{4}$ Virology Laboratory, Department of Primary Industries, Elizabeth Macarthur Agricultural Institute, Menangle, Australia.

*These authors contributed equally to this work.
} 